\title{
Management of digital transformation of industrial enterprises based on maturity models
}

\author{
L. A. Malysheva ${ }^{1 *}, O$. G. Kharlamova ${ }^{2}$
}

${ }^{1}$ Bussiness School of Ural Federal University, professor, Doctor of Economics, Russia

${ }^{2}$ Bussiness School of Ural Federal University, senior lecturer, Russia

\begin{abstract}
This paper considers the issues of the implementation of the digital transformation of industrial enterprises: the analysis of the existing model (AS IS) and planning the desired model (TO BE), depending on different conditions. The authors present the existing business models of digital transformation, reveal different approaches to classification, as well as their drawbacks from the point of view of practical application. In general, the models can be applied to diagnostics, but not to planning the desired state. The principle of classification of transformation models based on the life cycle of the market is proposed: monopoly, oligopoly, competition and monopsony. Firstly, the life-cycle approach allowed applying the author's Dynamic Model of Changes in Corporate Strategies (Dynamics) to digital transformation. This model was proposed earlier for the classification of traditional business models. Secondly, the life-cycle approach allowed using the maturity models of the industry, strategies, product, processes, data etc. in order to build a planning algorithm for the desired business model. As a result of the lifecycle approach to the classification of business models, it was possible to develop an algorithm for diagnosing and planning the desired digital transformation model, taking into account the limitations of maturity levels and present it in the form of a Digital Dynamic Model of Corporate Strategy Changes (Dynamics).
\end{abstract}

\section{Review of existing approaches to digital transformation}

The process of digital transformation of companies is becoming more and more popular and relevant. Different researchers interpret this term differently. A. Prokhorov and L. Konik note the differences in understanding by business consultants, engineers and developers, personnel specialists [1]. There are clearly three approaches to digital transformation: 1) strategic, associated with changing business models; 2) technological, related to automation, data-based decision making; 3) cultural, associated with new thinking. The authors also highlight the areas where the changes take place. These are: strategy, technology, people and processes.

We believe that all three aspects have a cause-effect relation. In the course of digital transformation, strategies, business models, technologies, business processes, ways of storing data, people's thinking, and their behavior are changing. This is not a complete list of system elements subject to transformation.

In the report of NRU HSE it is noted that the proliferation of new business models significantly reduce the time of market launch of new products. These models are characterized by an iterative approach to meet customer needs [2]. The authors look at the process of transformation from the perspective of business models that are changing the way we make money. They distinguish the following categories of business models: 1) digital platforms; 2) service business models (SaaS Software-as-a-Service); 3) product models (PaaS Product-as-a-Service); 4) crowdsourcing, 5) monetization of personal data, etc.

A similar view of the transformation of business models is given in the article by $\mathrm{P}$. Bilenko, $\mathrm{Yu}$. Krasilnikova and S. Nochovny [3]. The authors consider 6 prerequisites for the emergence of new business models in the context of the transformation of industrial enterprises: 1) the increasing complexity and fragmentation of the supply network; 2) decentralization; 3) network effects of online platforms; 4) democratization of design and prototyping; 5) mass customization; 6) sustainable development trend.

The same authors identify 7 promising business models for digital transformation of enterprises and also emphasize the combination of models.

1. MaaS / XaaS (Manufacturing-as-a-Service / Anything-as-a-Service). The provision of value chain elements as services or service packages. Online platforms that aggregate requests from customers and place orders with partners and subcontractors

2. C2M-model (Customer-to-Manufacturer or Community-to-Manufacturer). Interaction of 
factories with the end consumer. Personalization and customization of orders

3. Experimental crowdsourcing. Engagement of communities and experts to test and refine a product

4. Distributed factories. They are based on decentralization and suppose networking of ecommerce micro-shops

5. Technological hubs. Networking of project teams with experts, investors, educational organizations, factories and other ecosystem participants within the framework of high-tech accelerators

6. Selling software and monetizing data. Provision of value-added services based on data

7. Processing and reprocessing. Upgrading existing processes by modular upgrading of value chain elements.

In our opinion, the list of models characterizes the current situation, but it has a number of methodological shortcomings: it does not have completeness, mutual exclusion and clear grounds for classification. The reasoning at the level of examples of business models makes it possible to see development options, but does not provide an algorithm to choose an acceptable option and does not allow analyzing, planning and making decisions. We see two scientific challenges that will be discussed in this paper.

1. The classification of business models appropriate for making decisions on the preferred business model and suitable for diagnosing the current state and designing the desired one.

2. Algorithm for diagnostics and design of the desired state.

\section{Classification of business models of digital transformation}

Basic ideas:

1. The classification of business models should be based on the external conditions. The reasons for the change in the business model lie in the external conditions and a company behavior is a consequence of the influence of the external conditions and the result of the adaptation of a company

2. The classification of business models allows not only analyzing the existing state of a company, predicting the desired and ideal state of a company, but also developing a diagnostic and design algorithm

The purpose of the study is to propose a classification of the business models of a company, appropriate for both analysis and design of an ideal model

The proposed methodology is based on the Dynamic Model of Changing Corporate Strategies - Dynamics developed by the authors, which previously structured traditional business models [4]. This paper provides the extension of the DinAMIX model for digital business models.

\subsection{Review of existing approaches to the classification of business models of digital transformation}

One of the popular approaches to the classification of business models was proposed by Peter Wyle and Stephanie Warner [5]. The structuring is based on 2 principles: complete/incomplete knowledge of a client and the structure of business: linear, based on the value chain, or network, ecosystem.

According to the combination of factors, the authors determine 4 business models:

1. A supplier who sells his product through other companies, for example, marketplaces.

2. Omnichannel: a complex value chain that brings together various players in the market.

3. A modular manufacturer of finished products or services.

4. Ecosystem driver.

We see some limitations in the applicability of this classification, for example, in the absence of clear criteria to refer a company to one category. The second difficulty is in the fact that the basis of classification does not take into account external reasons for the division of companies, but internal factors that are a consequence.

Another classification is offered by Carsten Linz, Gunther Müller-Stevens and Alexander Zimmerman [6]. Their approach is based on the differences in transaction coverage and level of customization. Transaction Coverage: low, single or high, complex, integrative. Customization level is low, standardized, or highly customized.

Depending on the combination of factors, the authors determine 4 business models: product-oriented; platform-oriented; project-oriented and solutionoriented.

In our opinion, both the first and the second approaches are similar and quite correctly describe the behavior of companies. However, they do not give an answer to the question of the reasons for the changes, the limits of applicability, as well as clear criteria to refer a company to one type of business model.

The publications by A. Soolyatte [7], A. Kulizhsky [8], V. Kotelnikov [9], B. Garifullin and V. Zyablikov [10], Gassman O., Frankerberger K., Shik M. [ 11] and many other researchers are devoted to the classification of business-models.

In general, we are talking about the ways to monetize and analyze the behavior of companies. However the authors do not provide an answer to the question of the choice of an effective business model in each specific situation. Therefore, the task of the choice of a business model remains unresolved.

We believe that the choice of a business model should be based on external factors, for example, the type of market determined by the ratio of supply and demand.

In our previous works, we introduced a classification of traditional business models based on supply and demand in the author's business model DynaMIX. At the beginning of the cycle, demand exceeds supply, which 
corresponds to a small number of companies in the market under the conditions of monopoly. Then, as the market develops and the number of players increases, price fixing appears and an attempt to minimize costs due to transaction costs. Then the market becomes saturated and competitive. Finally, it degenerates into monopsony, the buyer market. Depending on this, 4 situations are determined that require different strategies and behavior of companies. These are: a monopoly characterized by growing demand; oligopoly with stable demand; competition and monopsony, with a significant excess of supply relative to demand.

Different types of markets allow talking about different strategies, types of products, business processes, structures, incentive systems for traditional models. Markets are also relevant when applied to digital transformation. We can use the same approach for digitalization conditions and consider the change in the market situation as a reason, and strategies, product type, business processes, information systems, data storage methods - as the areas of change. In the process of digitalization, data, methods of storing and processing them as well as the methods of decision-making become the most important. It is this area that significantly distinguishes digital transformation business models from traditional ones.

\subsection{Classification of business models taking into account the market life cycle and maturity models}

The lifecycle approach to classification allows developing discrete system states for each type of market.

At each stage, we recommend that companies choose different strategies, product types and, accordingly, behavior. It is a consequence of changes in the market and the adaptability of a company in certain areas: strategy, product, business processes, data collection and processing, decision-making algorithms. We classified business models by market type (Table 1).

Table 1. Classification of digital transformation business models by market type

\begin{tabular}{|l|l|l|l|l|}
\hline \multicolumn{1}{|c|}{ Type of market } & \multicolumn{1}{c|}{ Monopoly } & \multicolumn{1}{c|}{ Oligopoly } & \multicolumn{1}{c|}{ Competition } & \multicolumn{1}{c|}{ Monopsony } \\
\hline Supply and demand ratio & Demand $>>$ Supply & Demand $>$ Supply & Demand <Supply & Demand << Supply \\
\hline Strategy & Production growth & Reduced costs & Differentiation & Focusing \\
\hline Type of product & Typical, standard & Cost optimized & Modular & Service on request \\
\hline Model by P. Vail and S. Warner & Provider & $\begin{array}{l}\text { Modular } \\
\text { manufacturer }\end{array}$ & Omnichannel & Ecosystem driver \\
\hline $\begin{array}{l}\text { Model by K. Linz, G. Müller- } \\
\text { Stevens, A. Zimmermann }\end{array}$ & Product & Platform & Project & Decision \\
\hline
\end{tabular}

Under the conditions of monopoly, it is profitable for companies to increase production volumes through product standardization. They act as a supplier of a typical product and can post information about it on marketplaces. The focus is on product standardization. With the transition to oligopoly, products with a lower price appear which necessitates a change in strategy in favor of cost reduction in order to maintain the same rate of return. Companies can offer modular solutions for placement on the platform and connect with their module to the value chain for a client, based on minimizing the cost of non-core functions.

In competitive conditions, it is useful for a company to place its offers on the platform and use seamless integration (omnichannel) with partners and suppliers for flexible customization of orders from a consumer. Moreover, every order will be a project, since it will differ in the set of modules, resources and costs. Finally, in a monopsony conditions, ecosystems consisting of many services and providing focus on consumer needs through a variety of services and the ability to fulfill a personalized request will be effective.

The Life Cycle concept is closely related to maturity models. Market maturity, business processes and structures are also present here. However, waiting for industrial enterprises, we will be interested in technological models of maturity to a greater extent, which become primary in the context of digitalization.
There are many maturity models and they are applied to different types of companies and functional areas, for example:

- data maturity model (DMM) - for companies engaged in data processing and commercializing data as a product [13];

- analytic maturity model of a company (A2CM2) for companies specializing in forecasting and analytical data processing [14]

- model of technological readiness for commercialization (TRL) - for technology companies and those engaged in research and development (R\&D - Research \& Development) [15]

- capability maturity model integration (CMMI) - for companies with digital products [16].

We suppose that the abundance of maturity models is reasoned by the fact that at a given level of market development, different companies specialize in certain methods of data processing, analytics and research. In future, companies will expand their potential through the integration into ecosystem solutions.

Maturity models are used for diagnostics. They have categories and a scale for assessment, quantitatively specifying the ideal model and the current one.

The Data Maturity Model (DMM) has 6 segments: 1) Strategic dynamics; 2) Customer focus; 3) Digital culture, talents and skills; 4) Innovation and lean 
delivery; 5) Big data and artificial intelligence; 6) Technological leadership.

Each segment has its own subsections and weights, which depend on the type of company, in accordance with the classification of P. Vail and S. Warner. It is a supplier, modular manufacturer, omnichannel, ecosystem driver. We set the types of a company in accordance with the types of the market, taking into account the life cycle approach.

The subsections of the DMM are divided into categories and described. It is proposed to evaluate indicators on a 10-point scale, while the methodology provides a description of the best indicator value of 10 points. Depending on the final result, the following levels of maturity are distinguished: initial, controlled, definite, quantitatively controlled and optimal.

The Data Maturity Model (DMM) is good for diagnosis, but not good for planning, for the following reasons:

- intuitive choice of the type of company by the respondents, in accordance with the methodology of P. Vail and S. Warner;

- the multi-product nature of production enterprises, which does not allow a company to be classified as one type;

- the absence of a logical planning algorithm in the maturity model, which is not important in diagnostics, but critical in design.

In addition, as the market changes and the need to choose a more mature business model, a company will face the technological limitations of the existing data model.

In order to implement digital transformation, we propose to structure DynaMIX, taking into account maturity models, according to the following segments: market type, strategy, product type, type of business process, data model (DMM). For each product in the product portfolio, the desired model can be designed offline.

The proposed classification of business models based on market types (Dynamics) has the following features:

1. It considers each product in the product portfolio separately. At the same time, with one product a company can act as a supplier, and with another it can be a modular manufacturer. Dividing products by market type makes it possible to choose more clearly the type of business model.

2. It matches the type of business model to the type of market, and also structures the levels of maturity of the potential of a company in relation to the life cycle of the market, product, business processes, data and digital culture.

3. It evaluates not the average level of maturity of the potential of a company, but detailed one for different products.

Thus, we obtain the structure of the maturity model, weight coefficients for structural elements and scales to assess the maturity level of factors of structural elements. According to the scale, we can determine the ideal state of the business model, ideal in each situational range, as well as the limits of applicability of the weights.

A business model based on maturity models is used for analysis (AS IS) and planning (TO BE), taking into account external factors, for example, the market situation. The implementation of the changes will require a transition from the existing business model to the desired one.

\section{Classification results and algorithm to design a digital business model}

In the course of digital transformation most companies use the services of vendors and consultants who offer their own solutions. The decisions are related to business models, however, companies still do not distinguish between traditional and digital models, relying on the popular business model of A. Osterwalder in the description [17].

Vendor solutions often do not provide a systemic vision and solve current problems without the compliance with a long-term strategy. Maturity models, in turn, do not cover all functional areas requiring changes. They do not provide a logical and systematic view of changes.

The use of DynaMIX as a logical foundation allows building logic of changes similar to changes in traditional models.

The changes in the market situation necessitate internal changes and adaptation of a company to environmental factors. The sequence of changes in the course of digital transformation is as follows:

1) Product strategy;

2) Product type;

3) Value proposition;

4) Type of behavior;

5) Business processes;

6) Data (DMM).

After the optimization of business processes, the organizational structure and employee motivation system are adjusted in traditional models.

In digital transformation, the Data Maturity Model (DMM) is important. The authors of the model do not provide a logical sequence for the analysis of the maturity level. During the process of planning, consistency is extremely important.

We propose the following sequence for the design of the desired DMM model:

1) Customer focus;

2) Technological leadership;

3) Innovation and Lean Manufacturing;

4) Big data and artificial intelligence;

5) Digital culture, talents and skills;

6) Strategic dynamics.

The logic is as follows. According to the type of market, a company chooses a different level of customer focus and type of a product. Each type of product requires a certain level of technological leadership and there is a certain connection to business processes. Innovation and lean production are also business 
processes, eliminating waste and inefficient functions. The processes generate data, thus the next step is to design a data model in the category of Big Data and Artificial Intelligence. For this, digital culture, talents and skills are important. The plan of development and training is drawn up. Finally, strategic dynamics are assessed and compared with the initially chosen strategy.

The Digital Dynamic Model of Changing Corporate Strategies was called DynaMIX - Digital.
We summarized DynaMIX in a table, while the planning algorithm was located in the left column of the table, the classifier of the model states was set by the types of markets, and, in fact, the business models themselves were arranged in columns, in relation to the types of markets. The calculations in the DMM model were made in accordance with the ODI - Open Data Institute methodology (Table 2) [18].

Table 2. Algorithm for the design of the desired business model based on DynaMIX

\begin{tabular}{|l|c|c|c|c|}
\hline \multicolumn{1}{|c|}{ Type of market } & Monopoly & Oligopoly & Competition & Monopsony \\
\hline Demand/Supply & D>> S & D>> S & D > S & D>>S \\
\hline Strategy & Production growth & Reduced costs & Differentiation & Focusing \\
\hline Type of product & Typical, standard & Modular, typical & Modular on request & Service on request \\
\hline $\begin{array}{l}\text { Value proposition according to the model } \\
\text { of K. Linz, G. Müller-Stevens, A. } \\
\text { Zimmermann }\end{array}$ & Product & Platform & Project & Decision \\
\hline $\begin{array}{l}\text { Behavior type: the model of P. Vail and } \\
\text { S. Warner }\end{array}$ & & & & \\
\hline Business processes & Provider & $\begin{array}{c}\text { Modular } \\
\text { mManufacturer }\end{array}$ & Omnichannel & Ecosystem driver \\
\hline$\quad$ DMM & BPM & BPR/BPI & BR & XE \\
\hline 1. Customer focus & $\mathbf{7 , 2 7}$ & $\mathbf{9 , 1 2}$ & $\mathbf{9 , 5 0}$ & $\mathbf{1 0}$ \\
\hline 2. Technological leadership & 5,54 & 7,79 & 9,91 & 10 \\
\hline 3. Innovation and Lean Manufacturing & 7,65 & 8,62 & 9,65 & 10 \\
\hline 4. Big data and artificial intelligence & 7,78 & 10,00 & 9,06 & 10 \\
\hline 5. Digital culture, talents and skills & 8,72 & 9,43 & 9,47 & 10 \\
\hline 6. Strategic dynamics & 8,62 & 9,87 & 9,82 & 10 \\
\hline
\end{tabular}

DDynaMIX and DynaMIX include traditional concepts of business process management. They do not change for digital models.

- BPM - Business Process Management ;

- BPR - Business Process Reengineering;

- BPI - Business Process Improvement;

- $\quad$ BR - Business Reengineering;

- $\mathrm{XE}$ - Cross Engineering - reengineering without limits (companies).

The data maturity model (DMM) calculates the ideal maturity level for each segment for each type of company, taking into account the weights of the questions and the recommended values.

The imposition of a data maturity model (DMM) on the market lifecycle allowed drawing the following conclusions.

1. Depending on the level of market maturity, the type of company becomes more complex, and with it the level of data maturity increases quantitatively.

2. Under the conditions of monopoly, the level of client orientation has the lowest status, as well as the values of all other categories. Indeed, the business of a company model in this situation is the simplest and it does not require special strategic dynamics.

3. Under the conditions of oligopoly, the maximum weight is assigned to the category "Innovation and Lean Manufacturing". This is explained by the strategy to reduce costs and optimize business processes.
4. Under the conditions of competition, a company chooses a differentiation strategy. It is customized. It provides a buyer with the opportunity to choose a product from different modules, based on a seamlessly assembled value chain. This situation corresponds to a high level of customer focus, a lower level of innovation and lean manufacturing due to a focus on product quality rather than costs. There is also a reduced level of strategic dynamics and digital culture, talent and skills due to possible integration with other suppliers and subcontractors.

5. Under the conditions of monopsony, the level of maturity in all categories is maximal.

\section{Research results}

During the transition to digital transformation, traditional business models are no longer valid. It is necessary to rely on best practices and descriptive business models. At the same time, maturity models are developed, which are actively used to assess the readiness for digital transformation. However, maturity models are poorly applicable to planning, since they are not related to the cause of changes, strategies, necessary elements of the system and also do not provide the algorithm for the design of the desired model.

Based on the research results, we propose the following:

1. To classify digital models by market type, continuing the logic of DynaMIX and ensuring the 
continuity of business models. This approach became possible due to the underlying reason for any change - the market situation.

2. To supplement the Dynamic Model of Changing Corporate Strategies - DynaMIX with a data maturity model (DMM). It will make their combination appropriate for planning digital transformation, due to the interface with the external environment. We called it the generalized business model DynaMIX (Digital).

3. To develop a logical algorithm for the design of digital transformation based on the data maturity model (DMM). In this case, it is necessary to focus on the ideal correspondence of the model values to the types of companies.

\section{Conclusion}

The proposed Digital Dynamic Model of Changes in Corporate Strategies DynaMIX in terms of the data maturity model is focused on the entire company, and in terms of strategy, product type and business processes it is focused on each product separately.

The Data Maturity Level (DML) is a limit for the operation in different digital markets. A company can only choose the type of behavior based on the level of maturity of the data.

We propose to consider digital transformation as a sequential set of innovations: management, product and organizational ones. Today the digitalization of products and processes is popular. However, companies will not get a systemic effect without a strategy and organizational transformation.

Further studies of the digital transformation of industrial enterprises will reveal industry-specific features, implementation difficulties and classify successful practices and implementation algorithms.

\section{References}

1. A. Prokhorov, L. Konik Digital transformation. Analysis, trends, world experience. (Moscow: "ComNewsGroup", 2019).

2. G. I. Abdrakhmanova, K. O. Vishnevsky, L. M. Gokhberg What is the digital economy? Trends, competencies, measurement of Ch-80, XX Apr. International scientific conference on the problems of economic and social development, Moscow, April 9-12. (2019)

3. P. Bilenko, Y. Krasilnikova, S. Nochovny Factories in containers, MaaS-platforms and local micro-shops: the main production business models of the 2020s. Retrieved from: https://trends.rbc.ru/trends/innovation/6087d8629 a7947e710a41556

4. L.A. Malysheva Controlling Organizational Change: How Not to Drown in a Sea of Popular
Concepts? Tutorial. (Yekaterinburg: Educational and Methodological Center of USTU-UPI. 2010),

5. P. Vail, S. Warner, Digital business transformation: Changing the business model for a new generation of organizations (Moscow: Alpina Publisher, 2019).

6. C. Linz, G. Müller-Stephen, A Zimmerman, Radical change in the business model: Adaptation and survival in a competitive environment (Moscow: Alpina Publisher, 2019)

7. A. Soolyatte Business models of companies: definition, evolution, classification. Retrieved from:

http://www.klubok.net/article2302.html/2009/30/ 01

8. Kulizhsky A. Global competition of business models. How to choose and build your own? Retrieved from: http://www.executive.ru/community/articles/7892 220/2008/01/07

9. V. Yu. Kotelnikov New business models for a new era of rapid change driven by innovation (Moscow: Eksmo, 2007)

10. B.M. Garifullin, V.V. Zyabrikov Creative Economy. 13 (1), 83-92 (2019). doi: 10.18334 / ce.13.1.39720

11. O. Gassman, K. Frankerberger, M. Schick, Business models: 55 best templates. (Moscow: Alpina Publisher, 2016).

12. E. Borisov, A. Minov, B. Slavin Digital transformation of business models Retrieved from: 4cio.ru >content / 4CDTO / bori.pdf

13. CMMI - Capability Maturity Model Integration Retrieved from: https://www.tutorialspoint.com/cmmi/cmmioverview.htm

14. TRL - Technology Readiness Levels Retrieved from:

https://www.economy.gov.ru/material/file/53525 6e8193016b438e70338a9a0d42d/metodic_ta_mrg .pdf

15. Model of maturity of analytical abilities of the company Advanced Analytics Capability Maturity Model (A2CM2) Retrieved from: https://www.ssa.gov/data/data_governance_board /ACE_A2CM2_for\%20DGB.pptx.pdf

16. Model of maturity of data (DMM - Data Maturity Model). $\quad$ Retrieved from: https:/github.com/SuperNerb/Data-MaturityModels

17. A. Osterwalder, I. Pigne. Building business models (Moscow: Alpina Publisher, Skolkovo Series, 2013).

18. Methodology for calculating the level of maturity of data Retrieved from: https://theodi.org/article/open-data-maturitymodel-2/ 